\title{
Multiple-time higher-order perturbation analysis of the regularized long-wavelength equation
}

\author{
R. A. Kraenkel, ${ }^{1}$ M. A. Manna, ${ }^{2}$ V. Merle, ${ }^{2}$ J. C. Montero, ${ }^{1}$ and J. G. Pereira ${ }^{1}$ \\ ${ }^{1}$ Instituto de Física Teórica, Universidade Estadual Paulista, Rua Pamplona 145, 01405-900 São Paulo, Brazil \\ ${ }^{2}$ Physique Mathématique et Théorique, URA-CNRS 768, Université de Montpellier II, 34095 Montpellier Cedex 05, France
}

(Received 15 April 1996)

\begin{abstract}
By considering the long-wavelength limit of the regularized long wave (RLW) equation, we study its multiple-time higher-order evolution equations. As a first result, the equations of the Korteweg-de Vries hierarchy are shown to play a crucial role in providing a secularity-free perturbation theory in the specific case of a solitary-wave solution. Then, as a consequence, we show that the related perturbative series can be summed and gives exactly the solitary-wave solution of the RLW equation. Finally, some comments and considerations are made on the $\mathrm{N}$-soliton solution, as well as on the limitations of applicability of the multiplescale method in obtaining uniform perturbative series. [S1063-651X(96)08909-X]

PACS number(s): $03.40 . \mathrm{Kf}$
\end{abstract}

\section{INTRODUCTION}

The regularized long-wavelength (RLW) equation,

$$
u_{t}+u_{x}-u_{x x t}-6 u u_{x}=0 \text {, }
$$

also known as the Peregrine [1] or Benjamin-Bona-Mahony [2] equation, was originally introduced as an alternative for the Korteweg-de Vries ( $\mathrm{KdV}$ ) equation

$$
u_{t}+u_{x}+u_{x x x}-6 u u_{x}=0 \text {. }
$$

Despite having quite different dispersion properties, these two equations possess an intimate relationship. For example, the linear dispersion relation of the RLW equation is

$$
\omega(k)=\frac{k}{1+k^{2}},
$$

which, by the way, is the same as the dispersion relation of the shallow water wave equation [3]. For long wavelengths, $k$ is small, and $\omega(k)$ can be expanded according to

$$
\omega(k)=k-k^{3}+O\left(k^{5}\right) .
$$

The first two terms of this expansion coincide exactly with the complete linear dispersion relation of the $\mathrm{KdV}$ equation. Thus, for sufficiently long wavelengths, the traveling-wave solutions of Eqs. (1) and (2) are expected to be quite similar. Despite this, there is a deep difference between these two cases, since a polynomial is definitely not equivalent to an infinite series. This difference, which appears when higherorder terms of the dispersion relation expansion are considered, might also show up at higher-order approximations in a perturbation theory.

As is well known, the $\mathrm{KdV}$ equation governs the first relevant order of an asymptotic perturbation expansion, describing weakly nonlinear dispersive waves. However, to make sense of it as really governing such waves, the large time behavior of the perturbative series must be analyzed [4]. In other words, one needs to study the evolution equations of the higher-order terms of the perturbative expansion to check for the existence or not of secular-producing terms. This study, usually neglected in the derivation of the $\mathrm{KdV}$ equation, is essential to guarantee the uniformity of the perturbative expansion, thus rendering a real meaning to the $\mathrm{KdV}$ equation.

Motivated by the above considerations, in this paper we are going to apply a multiple-time version [5] of the reductive perturbation method to study long waves as governed by the RLW equation. As we are going to see, the KdV equation appears at the lowest relevant order of the perturbative scheme. Then, by assuming a solitary wave solution for the $\mathrm{KdV}$ equation, we consider higher-order approximations and show that the corresponding solitary-wave-related secularproducing terms can be eliminated from every order of the perturbative scheme. The equations of the $\mathrm{KdV}$ hierarchy, which appear as a consequence of natural compatibility conditions, are shown to play a crucial role in the process of eliminating the secular producing terms. Once a secularityfree perturbative series is obtained, we show that it may be summed to give the exact solitary wave solution of the RLW equation. We then close the paper with a discussion on the $N$-soliton case, as well as on the limitations of the multiple scale method.

\section{MULTIPLE-TIME FORMALISM}

To study the long-wavelength limit of the RLW equation, we put

$$
k=\epsilon \kappa,
$$

with $\epsilon$ a small parameter. In this limit, the dispersion relation (3) can be expanded as

$$
\omega(\kappa)=\epsilon \kappa-\epsilon^{3} \kappa^{3}+\epsilon^{5} \kappa^{5}-\epsilon^{7} \kappa^{7}+\cdots
$$

Accordingly, the solution of the corresponding linear RLW equation can be written in the form

$$
\begin{aligned}
u & =a \exp \{i[k x-\omega(k) t]\} \\
& \equiv a \exp \left\{i\left[\kappa \epsilon(x-t)+\epsilon^{3} \kappa^{3} t-\epsilon^{5} \kappa^{5} t+\epsilon^{7} \kappa^{7} t+\cdots\right]\right\}
\end{aligned}
$$


where $a$ is a constant. As given by this solution, we now define a slow space

$$
\xi=\epsilon(x-t),
$$

as well as an infinity of properly normalized slow time variables,

$$
\tau_{3}=\epsilon^{3} t ; \quad \tau_{5}=-\epsilon^{5} t, \quad \tau_{7}=\epsilon^{7} t, \text { etc. }
$$

Consequently, we have

$$
\frac{\partial}{\partial x}=\epsilon \frac{\partial}{\partial \xi},
$$

and

$$
\frac{\partial}{\partial t}=-\epsilon \frac{\partial}{\partial \xi}+\epsilon^{3} \frac{\partial}{\partial \tau_{3}}-\epsilon^{5} \frac{\partial}{\partial \tau_{5}}+\epsilon^{7} \frac{\partial}{\partial \tau_{7}}-\cdots
$$

It is important to note that the introduction of slow time variables normalized according to the dispersion relation expansion are such that they allow for an automatic elimination of the solitary-wave-related secular-producing terms appearing in the evolution equations for the higher-order terms of the wave field [6].

\section{PERTURBATION THEORY}

The perturbative scheme consists of making the expansion

$$
u \equiv \epsilon^{2} \hat{u}=\epsilon^{2}\left(u_{0}+\epsilon^{2} u_{2}+\epsilon^{4} u_{4}+\cdots\right),
$$

and substituting it, together with Eqs. (10) and (11), into the RLW equation (1). The result is the multiple-time equation

$$
\begin{aligned}
& \left(\epsilon^{3} \frac{\partial}{\partial \tau_{3}}-\epsilon^{5} \frac{\partial}{\partial \tau_{5}}+\cdots\right) \hat{u} \\
& \quad-\epsilon^{2} \frac{\partial^{2}}{\partial \xi^{2}}\left(-\epsilon \frac{\partial}{\partial \xi}+\epsilon^{3} \frac{\partial}{\partial \tau_{3}}-\epsilon^{5} \frac{\partial}{\partial \tau_{5}}+\cdots\right) \hat{u} \\
& \quad-3 \epsilon^{3} \frac{\partial}{\partial \xi}\left(u_{0}^{2}+2 \epsilon^{2} u_{0} u_{2}+\cdots\right)=0 .
\end{aligned}
$$

We proceed then to an order-by-order analysis of this equation.

At the lowest order, we obtain

$$
u_{0 \tau_{3}}=F_{3} \equiv-u_{0 \xi \xi \xi}+6 u_{0} u_{0 \xi}=0,
$$

which is the $\mathrm{KdV}$ equation. Introducing an operator $L$, whose action on any component $u_{n}$ is given by the linearized $\mathrm{KdV}$ operator

$$
L u_{n} \equiv u_{n \tau_{3}}+u_{n \xi \xi \xi}-6\left(u_{0} u_{n}\right)_{\xi},
$$

the KdV equation (14) can be rewritten in the form

$$
L u_{0}=-6 u_{0} u_{0 \xi} .
$$

Our interest in this paper is concerned with solitary waves. Thus we assume $u_{0}$ to be the solitary-wave solution of the $\mathrm{KdV}$ equation (14),

$$
u_{0}=-2 \kappa^{2} \operatorname{sech}^{2} \theta_{3},
$$

where $\theta_{3}=\kappa\left[\xi-4 \kappa^{2} \tau_{3}\right]$. In this case, Eq. (16) becomes

$$
L u_{0}=48 \kappa^{5} \operatorname{sech}^{4} \theta_{3} \tanh \theta_{3} .
$$

In the next order, we obtain the equation

$$
L u_{2}=u_{0 \tau_{3} \xi \xi}+u_{0 \tau_{5}} .
$$

The evolution of $u_{0}$ in the time $\tau_{3}$ is given by the $\mathrm{KdV}$ equation (14), but the evolution of $u_{0}$ in the time $\tau_{5}$ is not known up to this point. However, the multiple-time formalism introduces constraints which determine uniquely the evolution of $u_{0}$ in any higher-order time [5]. To see how this is possible, let us make the following considerations.

First, to have a well ordered perturbative scheme, we impose that each one of the equations describing the higherorder times evolution of $u_{0}$ be $\epsilon$ independent when passing from slow $\left(\kappa, u_{0}, \xi, \tau_{2 n+1}\right)$ to laboratory coordinates $(k, u, x, t)$. This will select all possible terms to appear in $u_{0 \tau_{2 n+1}}$. For instance, the evolution of $u_{0}$ in the time $\tau_{5}$ is restricted to be of the form

$$
u_{0 \tau_{5}}=\alpha_{5} u_{0(5 \xi)}+\beta_{5} u_{0} u_{0 \xi \xi \xi}+\gamma_{5} u_{0 \xi} u_{0 \xi \xi}+\delta_{5} u_{0}^{2} u_{0 \xi},
$$

where $\alpha_{5}, \beta_{5}, \gamma_{5}$, and $\delta_{5}$ are constants to be determined. Then, by imposing the natural (in the multiple-time formalism) compatibility condition

$$
\left(u_{0 \tau_{3}}\right)_{\tau_{5}}=\left(u_{0 \tau_{5}}\right)_{\tau_{3}},
$$

it is possible to determine the above constants in terms of $\alpha_{5}$, which is left as a free parameter. As it can be verified [5], the resulting equation is the fifth-order equation of the $\mathrm{KdV}$ hierarchy,

$$
u_{0 \tau_{5}}=F_{5} \equiv u_{0(5 \xi)}-10 u_{0} u_{0 \xi \xi \xi}-20 u_{0 \xi} u_{0 \xi \xi}+30 u_{0}^{2} u_{0 \xi} .
$$

The right-hand side of this equation would in principle appear multiplied by the free parameter $\alpha_{5}$, which would account for different possible normalizations of the time $\tau_{5}$. However, since we have already defined the slow time normalizations, this parameter was taken to be 1 , in order to have agreement with the normalizations introduced in Eq. (9). This is an important point since, as we have already stated, it allows for an automatic elimination of the solitarywave-related secular-producing terms appearing in the righthand side of Eq. (19). These terms, when $u_{0}$ is assumed to be a solitary wave of the KdV equation, are always of the form [7]

$$
u_{0[(2 n+1) \xi]}, n=0,1,2, \ldots .
$$

Thus, using Eqs. (14) and (22), respectively, to describe $u_{0 \tau_{3}}$ and $u_{0 \tau_{5}}$, Eq. (19) becomes 


$$
L u_{2}=-2 u_{0 \xi} u_{0 \xi \xi}-4 u_{0} u_{0 \xi \xi \xi}+30 u_{0}^{2} u_{0 \xi} .
$$

We notice in passing that the substitution of Eqs. (14) and (22), respectively, for $u_{0 \tau_{3}}$ and $u_{0 \tau_{5}}$, with properly normalized slow times, allowed for an automatically elimination of all solitary-wave-related secular-producing terms of Eq. (19). In fact, Eq. (24) does not present any secular-producing term anymore. Moreover, we see that at this order $u_{0}$ must satisfy simultaneously the first two equations of the KdV hierarchy, respectively, in the slow times $\tau_{3}$ and $\tau_{5}$. Introducing the general definition

$$
\theta_{2 n+1}=\kappa\left[\xi-4 \kappa^{2} \tau_{3}+16 \kappa^{4} \tau_{5}-\cdots+(-1)^{n}(2 \kappa)^{2 n} \tau_{2 n+1}\right],
$$

such a solitary wave is given by

$$
u_{0}=-2 \kappa^{2} \operatorname{sech}^{2} \theta_{5},
$$

and Eq. (24) becomes

$$
L u_{2}=192 \kappa^{7} \operatorname{sech}^{4} \theta_{5} \tanh \theta_{5} .
$$

Assuming a vanishing solution for the associated homogeneous equation, we can write the solution of this equation in the form

$$
u_{2}=4 \kappa^{2} u_{0},
$$

with $u_{0}$ given by (26).

We proceed then to the next order, where we obtain

$$
L u_{4}=-u_{0 \tau_{7}}-u_{0 \tau_{5} \xi \xi}+u_{2 \tau_{5}}+u_{2 \tau_{3} \xi \xi}+6 u_{2} u_{2 \xi} .
$$

Following the same scheme used above, we can use the compatibility condition

$$
\left(u_{0 \tau_{3}}\right)_{\tau_{7}}=\left(u_{0 \tau_{7}}\right)_{\tau_{3}}
$$

to obtain the evolution of $u_{0}$ in the time $\tau_{7}$. It is given by

$$
\begin{aligned}
u_{0 \tau_{7}}= & F_{7} \equiv-u_{0(7 \xi)}+14 u_{0} u_{0(5 \xi)}+42 u_{0 \xi} u_{0(4 \xi)} \\
& +140\left(\mu m_{0}\right)^{3} \mu_{0 \xi}+70 u_{0 \xi \xi} u_{0 \xi \xi \xi}-280 u_{0} u_{0 \xi} u_{0 \xi \xi} \\
& -70\left(u_{0 \xi}\right)^{3}-70 u_{0}{ }^{2} u_{0 \xi \xi \xi},
\end{aligned}
$$

which is exactly the seventh-order equation of the KdV hierarchy. At this order, therefore, the solitary wave must satisfy simultaneously the first three equations of the KdV hierarchy, respectively, in the times $\tau_{3}, \tau_{5}$, and $\tau_{7}$. This means that

$$
u_{0}=-2 \kappa^{2} \operatorname{sech}^{2} \theta_{7} .
$$

Now, by using Eq. (28) to express $u_{2}$, and the equations of the $\mathrm{KdV}$ hierarchy to express $u_{0 \tau_{7}}, u_{0 \tau_{5}}$, and $u_{0 \tau_{3}}$, all secular-producing terms of Eq. (29) are automatically eliminated. Then, substituting the solution (32), Eq. (29) becomes

$$
L u_{4}=768 \kappa^{9} \operatorname{sech}^{4} \theta_{7} \tanh \theta_{7} .
$$

Again, by assuming a vanishing solution for the associated homogeneous equation, the solution of this equation can be written as

$$
u_{4}=\left(4 \kappa^{2}\right)^{2} u_{0},
$$

with $u_{0}$ given now by Eq. (32).

This procedure can be repeated up to any higher order. In other words, we can use the compatibility condition

$$
\left(u_{0 \tau_{3}}\right)_{\tau_{2 n+1}}=\left(u_{0 \tau_{2 n+1}}\right)_{\tau_{3}}
$$

to obtain the evolution of $u_{0}$ in the time $\tau_{2 n+1}$, which will turn out to be the $(2 n+1)$ th equation of the KdV hierarchy. In this case, $u_{0}$ will represent a solitary wave satisfying simultaneously the first $n$ equations of the KdV hierarchy:

$$
u_{0}=-2 \kappa^{2} \operatorname{sech}^{2} \theta_{2 n+1} .
$$

The resulting secularity-free evolution equation at this order will be

$$
L u_{2 n}=3(4)^{n+2}(\kappa)^{2 n+5} \operatorname{sech}^{4} \theta_{2 n+3} \tanh \theta_{2 n+3} .
$$

Assuming a vanishing solution for the associated homogeneous equation, the solution to this equation can be written in the form

$$
u_{2 n}=\left(4 \kappa^{2}\right)^{n} u_{0},
$$

with $u_{0}$ given by Eq. (36). Extending this procedure $a d$ infinitum, $u_{0}$ will represent a solitary wave satisfying simultaneously all equations of the KdV hierarchy, and we obtain an exact solution for the RLW equation.

\section{RETURNING TO THE LABORATORY COORDINATES}

Let us take the solutions $u_{2 n}$ and substitute them in expansion (12). Putting $u_{0}$ in evidence, we obtain

$$
u=\epsilon^{2} u_{0}\left[1+4 \epsilon^{2} \kappa^{2}+16 \epsilon^{4} \kappa^{4}+64 \epsilon^{6} \kappa^{6}+\cdots\right] .
$$

Now, the above series can be summed:

$$
1+4 \epsilon^{2} \kappa^{2}+16 \epsilon^{4} \kappa^{4}+64 \epsilon^{6} \kappa^{6}+\cdots=\frac{1}{1-4 \epsilon^{2} \kappa^{2}} .
$$

Therefore, we obtain the RLW exact solution

$$
\begin{aligned}
u= & -\frac{2 \epsilon^{2} \kappa^{2}}{1-4 \epsilon^{2} \kappa^{2}} \operatorname{sech}^{2}\left[\kappa \xi-4 \kappa^{3} \tau_{3}+16 \kappa^{5} \tau_{5}\right. \\
& \left.-64 \kappa^{7} \tau_{7}+\cdots\right] .
\end{aligned}
$$

Then, by using Eqs. (5), (8), and (9), we can rewrite $u$ in terms of the laboratory coordinates $(k, x, t)$. The result is

$$
u=-\frac{2 k^{2}}{1-4 k^{2}} \operatorname{sech}^{2}\left[k x-k\left(1+4 k^{2}+16 k^{4}+64 k^{6}+\cdots\right) t\right] .
$$

Again using Eq. (40) with $\epsilon \kappa=k$, we finally obtain

$$
u=-a \operatorname{sech}^{2}\left[k\left(x-\frac{t}{1-4 k^{2}}\right)\right], \quad a=\frac{2 k^{2}}{1-4 k^{2}},
$$

which is the solitary wave solution of the RLW equation (1).

The RLW equation has another solution, given by 


$$
u=b \tanh ^{2}\left[k\left(x-\frac{t}{1+8 k^{2}}\right)\right], \quad b=\frac{2 k^{2}}{1+8 k^{2}} .
$$

In fact, it is easy to see that Eq. (1) is invariant under the transformation

$$
t^{\prime}=a^{-1} t, \quad x^{\prime}=x, \quad u^{\prime}=b-a u,
$$

where, if $u$ is given by Eq. (43), $u^{\prime}$ turns out to be the solution given by Eq. (44). By following the same procedure used to obtain the RLW solitary wave solution (43), it is also possible to use the multiple-time perturbative scheme to obtain solution (44). This is done by choosing

$$
u_{0}=2 \kappa^{2} \tanh ^{2}\left(\kappa \xi-8 \kappa^{3} \tau_{3}\right),
$$

instead of (17) as the solution for the KdV equation (14). As higher orders are reached, this $u_{0}$ is also required to satisfy the higher-order equations of the $\mathrm{KdV}$ hierarchy, which amounts to include dependences on the higher-order times $\tau_{5}, \tau_{7}$, etc. However, there is an important difference: the secular-producing term in each order of the perturbative scheme will come not only from the linear term, but from both the linear and nonlinear terms. As a consequence, the slow time normalizations obtained from the linear dispersion relation expansion will not be able to remove the secularproducing terms in this case. In other words, different slow time normalizations will be needed to obtain a secularity-free perturbative series. These normalizations can be easily found by properly choosing the free parameters left at each order of the perturbation scheme [6]. After doing that, we obtain the following perturbative series for $u$ :

$$
\begin{aligned}
u= & \epsilon^{2}\left[1-8 k^{2}+64 k^{4}-\cdots\right] \\
& \times \tanh ^{2}\left[k x-k\left(1-8 k^{2}+64 k^{4}-\cdots\right)\right] .
\end{aligned}
$$

As in the previous case, these series can be summed, resulting in

$$
u=\frac{2 k^{2}}{1+8 k^{2}} \tanh ^{2}\left[k\left(x-\frac{t}{1+8 k^{2}}\right)\right]
$$

which is the solution (44) of the RLW equation. As already stated, however, another slow time normalization is needed in this case to obtain a secularity-free perturbative series, which is different from that obtained from the dispersion relation expansion.

\section{STUDY OF THE APPLICABILITY OF THE MULTIPLE-SCALE METHOD}

The multiple-scale method is not always able to remove all the secular-producing terms of a perturbative series [8]. In some cases, nonintegrable effects may preclude the existence of uniform asymptotic expansions. Considering that the RLW is nonintegrable, the purpose of this section will be to make a brief discussion of how those effects appear in the higher-order terms of the perturbative series for the specific case of the RLW equation. The approach we are going to use is that developed by Kodama and Mikhailov [8].

Let us start by defining slow variables according to

$$
u=\epsilon v, \quad \xi=\epsilon^{1 / 2}(x-t), \quad \tau_{3}=\epsilon^{3 / 2} t .
$$

In these coordinates, and up to terms of order $\epsilon^{2}$, Eq. (1) becomes

$$
\begin{aligned}
v_{\tau_{3}}= & \partial_{\xi}\left[3 v^{2}-v_{\xi \xi}+\epsilon \partial_{\xi \xi}\left(3 v^{2}-v_{\xi \xi}\right)\right. \\
& \left.+\epsilon^{3} \partial_{(4 \xi)}\left(3 v^{2}-v_{\xi \xi}\right)+\cdots\right]
\end{aligned}
$$

Then, we make a near identity transformation [9] given by

$$
v=w+\epsilon \Phi(w)+\epsilon^{2} \Psi(w)+O\left(\epsilon^{3}\right),
$$

where, for reasons of scaling-weight invariance, the differential polinomials $\Phi$ and $\Psi$, which are allowed to be nonlocal, can involve only the following terms:

$$
\begin{gathered}
\Phi=\alpha w^{2}+\beta w_{\xi \xi}+\gamma w_{\xi} \partial^{-1} w, \\
\Psi=a w^{3}+b\left(w_{\xi}\right)^{2}+c w w_{\xi \xi}+d w_{(4 \xi)}+e w w_{\xi} \partial^{-1} w \\
+f w_{\xi} \partial^{-1}\left(w^{2}\right)+g w_{\xi \xi \xi} \partial^{-1} w+h w_{\xi \xi}\left(\partial^{-1} w\right)^{2} .
\end{gathered}
$$

Substituting into (50), we obtain

$$
w_{\tau_{3}}=K_{3}+\epsilon K_{5}+\epsilon^{2} K_{7}+\cdots
$$

with

$$
K_{3}=\partial_{\xi} M_{0},
$$

$$
\begin{gathered}
K_{5}=\partial_{\xi}\left(M_{1}+\partial_{\xi \xi} M_{0}\right)-\frac{\delta \Phi}{\delta w}\left(\partial_{\xi} M_{0}\right), \\
K_{7}=\partial_{\xi}\left(M_{2}+\partial_{\xi \xi} M_{1}+\partial_{(4 \xi)} M_{0}\right)-\frac{\delta \Psi}{\delta w}\left(\partial_{\xi} M_{0}\right) \\
-\frac{\delta \Phi}{\delta w}\left[\partial_{\xi}\left(M_{1}+\partial_{\xi \xi} M_{0}\right)-\frac{\delta \Phi}{\delta w}\left(\partial_{\xi} M_{0}\right)\right],
\end{gathered}
$$

where we have introduced the notation

$$
\begin{gathered}
M_{0}=3 w^{2}-w_{\xi \xi}, \\
M_{1}=6 w \Phi-\Phi_{\xi \xi}, \\
M_{2}=3 \Phi^{2}+6 w \Psi-\Psi_{\xi \xi} .
\end{gathered}
$$

At order $\epsilon^{0}$ we find

$$
K_{3}=F_{3} \equiv 6 w w_{\xi}-w_{\xi \xi \xi},
$$


that is, $K_{3}$ is the symmetry of order $\epsilon^{0}$ of the $\mathrm{KdV}$ equation. At the next order, by properly choosing $\alpha, \beta$, and $\gamma$, we find

$$
K_{5}=F_{5},
$$

with $F_{5}$ defined by Eq. (22). This means that there exists a near-identity transformation (51)-(53) such that $K_{5}$ is the symmetry of order $\epsilon$ of the KdV equation. In the first two orders, therefore, no problems appear. This is a general result that holds for any equation, not only for the particular case of the RLW equation. It is in the next order that the so called obstacles [8] show up. In fact in the next order we obtain

$$
K_{7}=F_{7}+\mathcal{O}(w),
$$

with $F_{7}$ defined by Eq. (31), and $\mathcal{O}(w)$ representing the obstacle, which is given by

$$
\begin{aligned}
\mathcal{O}(w)= & \left(-\frac{32}{3}-3 g\right) w w_{(5 \xi)}+\left(-\frac{20}{3}-3 c-24 d-3 g\right) w_{\xi} w_{(4 \xi)}+\left(-\frac{508}{3}+6 a+2 f-18 g\right) w^{3} w_{\xi} \\
& +(22-3 c-6 b-60 d) w_{\xi \xi} w_{\xi \xi \xi}+\left(\frac{700}{3}-18 a-12 c-6 f+72 g\right) w w_{\xi} w_{\xi \xi}+\left(\frac{224}{3}-3 f+21 g\right) w^{2} w_{\xi \xi \xi} \\
& +\left(\frac{158}{3}-6 a-6 b-3 f+18 g\right)\left(w_{\xi}\right)^{3} .
\end{aligned}
$$

The important point is that, for an arbitrary $\mathrm{KdV}$ hierarchy solution $w$, it is not possible to choose $a, b, \ldots, g$ in such a way to have a vanishing obstacle. However, as an explicit calculation easily shows, when $w$ is a solitary-wave solution of the $\mathrm{KdV}$ hierarchy, there is a near-identity transformation leading to $O(w)=0$.

The above considerations are important in the sense that they clarify the results obtained in the previous sections concerning solitary-wave-related secularities. But, at the same time, they put into evidence the limitations of the perturbative scheme which, as we now know, cannot be extended to the two-or-more soliton solutions in the nonintegrable case. On the other hand, for integrable systems, as for example the shallow water wave equation, the multiple-scale method will be able to handle both the solitary-wave- and the $\mathrm{N}$-soliton-related secularities [10] since no obstacles will be present in either case.

\section{FINAL REMARKS}

We have applied a multiple-time version of the reductive perturbation method to study the solitary-wave solution of the RLW equation. As it has already been shown [5], the use of multiple-time scales allows for the elimination of all solitary-wave-related secular-producing terms appearing in the evolution equations of the higher-order terms of the wave field. Moreover, it has also been shown [6] that these secularities are automatically removed if the slow time scales are normalized according to the long-wavelength expansion of the dispersion relation of the original equation. By using this strategy, we have succeeded in expressing the solitary wave solution of the RLW equation as a sum of solitary-waves satisfying simultaneously, in the slow coordinates, all equations of the KdV hierarchy. Similar results have been shown to hold also for the Boussinesq [11] and the shallow water wave [11] equations. However, while in these two cases the solitary wave solution was obtained due to a truncation of the perturbative series, the RLW solitary wave was obtained by summing the perturbative series.

To finish, let us make the following considerations. If we assume the RLW equation to be an exact model equation, as we have in fact done, the KdV equation appears as its longwavelength leading-order approximation. This is one more confirmation of the widely known property of the KdV equation, which states that it holds a unique, privileged, and universal meaning in the sense it appears as the leading-order approximation of any weakly nonlinear dispersive systems, as for example that represented by the RLW equation. From this point of view, the old dispute [12] on the equivalence of the RLW and the KdV equations would be made on a different ground, since the RLW equation should be compared not to the KdV equation, but to the whole set of equations of the KdV hierarchy. In other words, the RLW equation should be compared not to its leading-order approximation, but to the whole perturbative series. And according to our results, as far as solitary waves are concerned, the RLW equation is indeed equivalent to the $\mathrm{KdV}$ hierarchy, since a solitary wave of the RLW equation is nothing but an infinite series given by the sum of solitary waves satisfying simultaneously all equations of the KdV hierarchy, each one in a different slow time variable.

\section{ACKNOWLEDGMENTS}

The authors would like to thank J. Léon for useful discussions. They would also like to thank Y. Kodama and A. V. Mikhailov for sending the preprint of Ref. [8] to them. This work was partially supported by CNPq (Brazil) and CNRS (France). 
[1] D. H. Peregrine, J. Fluid Mech. 25, 321, (1966).

[2] T. B. Benjamin, J. L. Bona, and J. J. Mahony, Philos. Trans. R. Soc. London Ser. A 272, 47 (1972).

[3] M. J. Ablowitz, D. J. Kaup, A. C. Newell, and H. Segur, Stud. Appl. Math. 53, 249 (1974).

[4] Y. Kodama, in Nonlinear Water Waves, IUTAM Symposium, Tokyo, Japan, 1987, edited by K. Horikawa and H. Maruo (Springer-Verlag, Berlin, 1988).

[5] R. A. Kraenkel, M. A. Manna, and J. G. Pereira, J. Math. Phys. 36, 307 (1995).

[6] R. A. Kraenkel, M. A. Manna, J. C. Montero and J. G. Pereira, Proceedings of the Workshop Nonlinear Physics: Theory and Experiment, Gallipoli, Lecce, 1995 (World Scientific, Sin- gapore, 1995) (patt-sol/9509003).

[7] Y. Kodama and T. Taniuti, J. Phys. Soc. Jpn. 45, 298 (1978).

[8] Y. Kodama and A. V. Mikhailov, Obstacles to Asymptotic Integrability, (unpublished).

[9] Y. Kodama, Phys. Lett. A 107, 245 (1985).

[10] R. A. Kraenkel, M. A. Manna, J. C. Montero, and J. G. Pereira (unpublished) (patt-sol/9509001).

[11] R. A. Kraenkel, M. A. Manna, J. C. Montero, and J. G. Pereira, J. Math. Phys. 36, 6822 (1995).

[12] M. Kruskal, in Dynamical Systems, Theory and Applications, edited by J. Moser, Lecture Notes in Physics Vol. 38 (Springer-Verlag, Berlin, 1975). 